\title{
Minimal residual HIV viremia: verification of the Abbott Real-Time HIV-I assay sensitivity
}

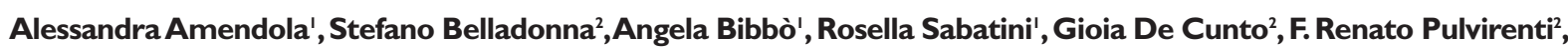
M. Rosaria Capobianchi'

I Laboratory of Virology INMI L. Spallanzani, Rome;

2 Abbott Molecular, Rome

Key words: HIV-I, Residual viremia, sensitivity, Real time

Viremia minima residua HIV: verifica della sensibilità del test Abbott Real-time HIV-I

\section{SUMMARY}

Introduction: In the HIV-I infection, the increase in number of CD4 T lymphocytes and the viral load decline are the main indicators of the effectiveness of antiretroviral therapy. On average, $85 \%$ of patients receiving effective treatment has a persistent suppression of plasma viral load below the detection limit ( $<50$ copies $/ \mathrm{mL}$ ) of clinically used viral load assays, regardless of treatment regimen in use. It is known, however, that, even when viremia is reduced below the sensitivity limit of current diagnostic assays, the virus persists in "reservoirs" and traces of free virions can be detected in plasma. There is a considerable interest to investigate the clinical significance of residual viremia. Advances in molecular diagnostics allows nowadays to couple a wide dynamic range to a high sensitivity. The Abbott Real-time HIV-I test is linear from 40 to $10^{7}$ copies $/ \mathrm{mL}$ and provides, below 40 copies $/ \mathrm{mL}$, additional information such as " $<40 \mathrm{cp} / \mathrm{mL}$, target detected" or "target not detected". The HIV-I detection is verified by the max-Ratio algorithm software. We assessed the test sensitivity when the qualitative response is considered as well.

Methods: A 'probit' analysis was performed using dilutions of the HIV-I RNA Working Reagent I for NAT assays (NIBSC code: 99/634), defined in IU/mL and different from that used by the manufacturer (VQA,Virology Quality Assurance Laboratory of the AIDS Clinical Trial Group) for standardization and definition of performances. The sample input volume $(0.6 \mathrm{~mL})$ was the same used in clinical routine. A total of 196 replicates at concentrations decreasing from 120 to 5 copies $/ \mathrm{mL}$, in three different sessions, have been tested. The 'probit' analysis (binomial dose-response model, 95\% "hit-rate") has been carried out on the SAS 9.I.3 software package.

Results: The sensitivity of the " $<40 \mathrm{cp} / \mathrm{mL}$, target detected" response was equal to 28,76 copies $/ \mathrm{mL}$, with $95 \%$ confidence limits between 22, 19 and 52,27 copies/mL.

Conclusions: With the protocol of $0.6 \mathrm{~mL}$, the Abbott RealTime test is able to detect viral concentrations down to 29 copies/mL. A further sensitivity increase below the current threshold is likely to be reached through the adoption of experimental protocols based on increased input volumes in order to improve significantly assay precision at low viremia level.

\section{INTRODUZIONE}

Nell'infezione da HIV-1, l’incremento del numero di linfociti T CD4 e il declino della carica virale sono i principali indicatori dell'efficacia della terapia antiretrovirale $(2,3)$. In media l'85\% dei pazienti sottoposti a terapia efficace mantiene una carica virale plasmatica persistente sotto il limite minimo (50 copie/mL) dei test utilizzati in clinica (4), indipendentemente dal regime terapeutico in uso. Tuttavia è noto che, anche quando la viremia scende al di sotto di 50 copie/mL, il virus persiste nei "reservoirs" e possono essere riscontrate nel plasma tracce di virioni liberi $(1,6)$.

Esiste un notevole interesse a quantificare la viremia residua e ad indagare sul suo significato clinico, in particolare nei regimi terapeutici altamente efficaci e nei nuovi protocolli sperimentali.

Grazie ai progressi compiuti nel campo della diagnostica molecolare, oggi esistono saggi in grado di misurare la carica virale con un ampio range dinamico ed una elevata sensibilità. Il test Abbott Real Time HIV-1 è lineare da 40 a $10^{7}$ copie/mL e fornisce, al di sotto di 40 copie $/ \mathrm{mL}$ (o $70 \mathrm{IU} / \mathrm{mL}$ ), informazioni aggiuntive di tipo " $<40$ copie/mL, target rilevato" o "target non rilevato", la cui autenticità è controllata dall'algoritmo software max-Ratio (5). Abbiamo verificato la sensibilità del metodo nel caso si consideri la risposta qualitativa “ $<40$ copie/mL, target rilevato" e analizzato la precisione del test per valori di viremia $<40$ copie/mL, non forniti dal metodo, ma da noi estrapolati dalla retta di calibrazione. L'attendibilità dei risultati così ottenuti è stata analizzata al fine di valutarne l'applicabilità negli studi sulla misurazione della viremia residua.

Scopo del lavoro è stato quello di verificare la sensibilità del metodo al di sotto del limite minimo di misurazione dichiarato (40 copie/mL) e valutare l'attendibilità dei risultati quan- titativi di viremia residua ottenuti per estrapolazione dalla retta di calibrazione.

\section{MATERIALI E METODI}

La sensibilità del metodo al di sotto del limite minimo di misurazione dichiarato $(40 \mathrm{cp} / \mathrm{mL})$ è stata analizzata utilizzando il $2^{\text {nd }} H I V-1$ RNA International Standard WHO (NIBSC code: 97/650) e il preparato HIV-1 RNA Working Reagent 1 for NAT Assays (NIBSC code: 99/634) a diluizioni scalari.

Il $2^{\text {nd }}$ HIV-1 RNA International Standard WHO (5,56 $\log _{10}$ IU) è stato diluito con plasma negativo a 30000, 3000, 1000, 300, 100, 30 e $10 \mathrm{IU} / \mathrm{mL}$ (10 repliche a 30000 e $3000 \mathrm{IU} / \mathrm{mL}$; 3 repliche a 1000, 300, 100, 30 e $10 \mathrm{IU} / \mathrm{mL}$ ).

L' HIV-1 RNA Working Reagent 1 for NAT Assays (3,56 $\log _{10} \mathrm{IU} / \mathrm{mL}$, pari a 2106 copie/mL con fattore di conversione dichiarato da Abbott) è stato diluito con plasma negativo a 80, 40, 20, 10 e 5 copie/mL (40 repliche per ciascuna diluizione). Il volume iniziale di campione analizzato è quello utilizzato nel protocollo di routine $(0,6 \mathrm{~mL})$. I risultati sono stati analizzati dopo trasformazione in $\log _{10}$. L'analisi 'probit' (modello dose-risposta binomiale, 95\% "hit-rate”) è stata condotta con il software SAS 9.1.3.

\section{RISULTATI}

Il sistema Abbott Real-Time HIV-1 quantifica il $2^{\text {nd }}$ International Standard HIV-1 RNA in IU/mL con accuratezza e precisione molto elevate (Figura I). La differenza media tra i valori attesi e i valori misurati è di $0,051 \pm 0,1 \log _{10}$ $\mathrm{IU} / \mathrm{mL}$ nel range lineare di misurazione. Se nella valutazione si aggiungono i valori misurati alle diluizioni a 30 e 10 $\mathrm{IU} / \mathrm{mL}$, la differenza media si mantiene a $0,11 \pm 0,13 \log _{10}$ IU/mL (Tabella 1).

\section{Corresponding author: Alessandra Amendola}

Laboratorio di Virologia - Istituto Nazionale per le Malattie Infettive (INMI) "Lazzaro Spallanzani”

00I49 Roma - Via Portuense 292 - Tel: 0655 I70 693 - Fax: 065594555

E-mail: alessandra.amendola@inmi.it 
È stata quindi condotta un'analisi 'probit' utilizzando diluizioni dell'HIV-1 RNA Working Reagent 1 for NAT Assays per la standardizzazione e la definizione delle prestazioni. Sono stati testati in totale 200 replicati a concentrazioni decrescenti da 80 a 5 copie/mL, in quattro differenti sedute. In figura II sono mostrati i valori misurati per ciascuna diluizione. I coefficienti di variazione misurati a ciascuna diluizione sono: 19,5\% (80 copie/mL), 13,6\% (40 copie/mL), 16,5\% (20 copie/mL), 19,2\% (10 copie/mL) e 13,1\% (5 copie/mL). La differenza media tra i valori attesi e i valori misurati è $0,14 \pm 0,31 \log _{10}$ copie/mL (Tabella 2$)$. L'analisi 'probit' dei risultati ottenuti con l'HIV-1 RNA Working Reagent 1 ha evidenziato che la sensibilita' della risposta “<40 copie/mL, target detected”, espressa come frequenza di rilevazione al 95\%, è 28,76 copie/mL (con limiti di confidenza al 95\%: 22,19-52,27) (Figura III).

Un ulteriore incremento della sensibilità del test è ottenibile concentrando 3-4 volte i campioni di plasma. Concentrando 4 volumi di campione (e precisamente $0,6 \mathrm{~mL}$ di plasma x 4 : pari in totale a 2,4 mL) mediante ultracentrifugazione a $20000 \mathrm{rpm}$, a $4^{\circ} \mathrm{C}$, per 90 minuti, e utilizzando il normale protocollo di routine (da 0,6 $\mathrm{mL}$ dopo aver aspirato il plasma in eccesso), si possono misurare i valori di viremia residua nei campioni risultanti $<40 \mathrm{copie} / \mathrm{mL}$, con una riduzione di carica virale dovuta alla procedura di concentrazione non superiore al $15 \%$ della carica stimata per mL iniziale.

\section{DISCUSSIONE E CONCLUSIONI}

Il sistema Abbott Real-Time è un sistema diagnostico accurato e preciso. Con il protocollo da $0,6 \mathrm{~mL}$, il saggio è in grado di rilevare concentrazioni virali fino a 29 copie/mL con una affidabilità al $95 \%$.

Un ulteriore incremento della sensibilità del test al di sotto dell'attuale soglia sarà probabilmente raggiungibile attraverso l'adozione di protocolli sperimentali basati su volumi input maggiori, per esempio concentrando i campioni, e attraverso il miglioramento della precisione del test a bassi valori di viremia.

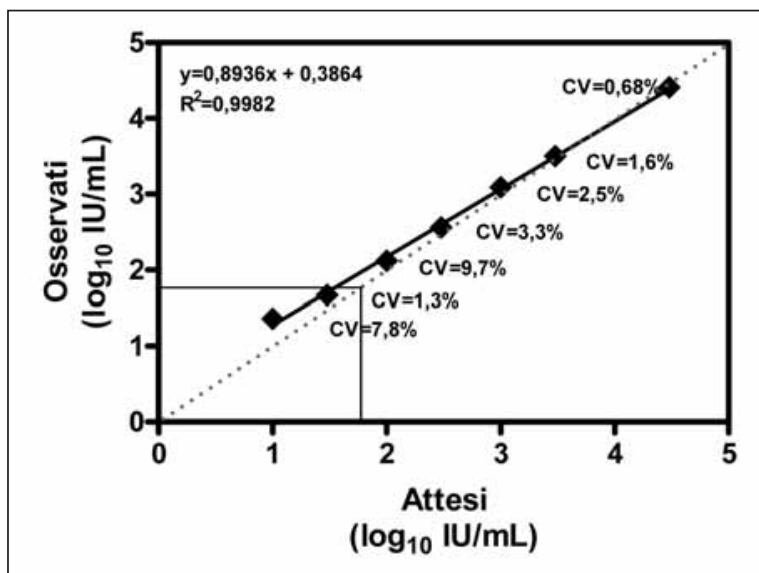

Figura I: $2^{\text {nd }}$ HIV-I RNA International Standard WHO (NIBSC code 97/650):Accuratezza, Linearità, Precisione.

Tabella I: $2^{\text {nd }}$ HIV-I RNA International Standard WHO (NIBSC code 971650):Valori attesi e misurati.

\begin{tabular}{ccc}
\hline $\begin{array}{c}\text { Atteso } \\
\left(\log _{1} \mid \mathrm{U} / \mathrm{mL}\right)\end{array}$ & $\begin{array}{c}\text { Misurato } \\
\left(\log _{10} \mid \mathrm{U} / \mathrm{mL}\right)\end{array}$ & $\begin{array}{c}\text { Differenza media } \\
(\text { Misurato-Atteso })\end{array}$ \\
\hline 4,48 & 4,41 & $-0,07$ \\
\hline 3,48 & 3,50 & 0,02 \\
\hline 3,00 & 3,09 & 0,09 \\
\hline 2,48 & 2,56 & 0,08 \\
\hline 2,00 & 2,12 & 0,12 \\
\hline 1,48 & 1,67 & 0,19 \\
\hline 1,00 & 1,36 & 0,36
\end{tabular}

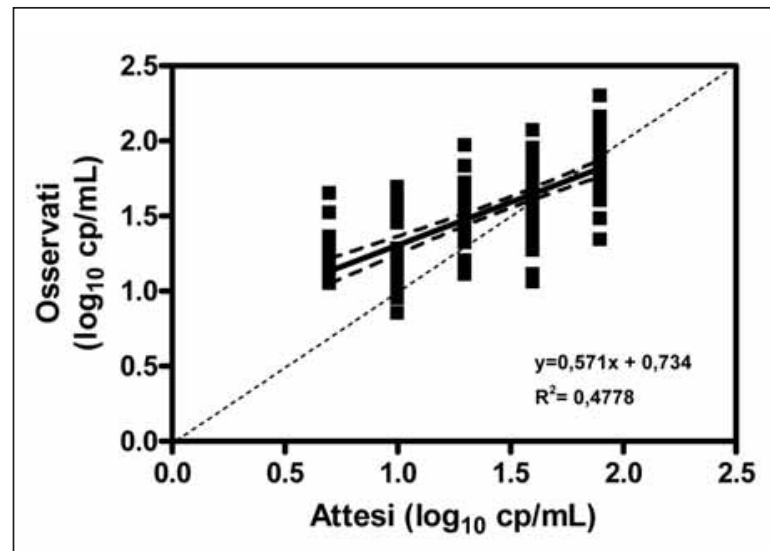

Figura II: HIV-I RNA Working Reagent I for NAT assays (NIBSC code 99/634):Valori attesi e osservati.

Tabella 2: HIV-I RNA Working Reagent I for NAT assays (NIBSC code 99/634):Valori attesi e misurati.

\begin{tabular}{ccc}
\hline $\begin{array}{c}\text { Atteso } \\
\left(\log _{10} \mathrm{cp} / \mathrm{mL}\right)\end{array}$ & $\begin{array}{c}\text { Misurato } \\
\left(\log _{10} \mathrm{cp} / \mathrm{mL}\right)\end{array}$ & $\begin{array}{c}\text { Differenza media } \\
\text { (Misurato-Atteso) }\end{array}$ \\
\hline $\mathrm{I}, 84$ & $\mathrm{I}, 57$ & $-0,27$ \\
\hline $\mathrm{I}, 60$ & $\mathrm{I}, 63$ & 0,03 \\
\hline $\mathrm{I}, 30$ & $\mathrm{I}, 40$ & 0,10 \\
\hline $\mathrm{I}, 00$ & $\mathrm{I}, 27$ & 0,27 \\
\hline 0,70 & $\mathrm{I}, 28$ & 0,58 \\
\hline
\end{tabular}

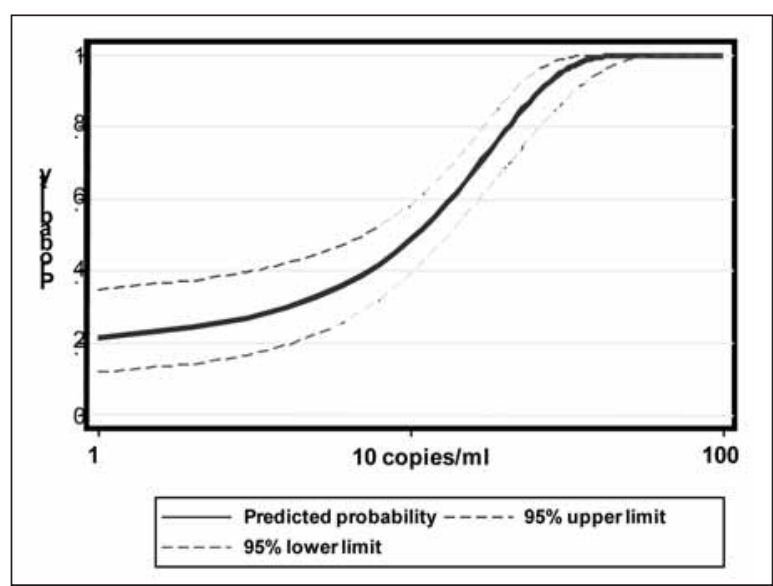

Figura III: Analisi Probit con HIV-I RNA Working Reagent I for NAT assays (NIBSC code 99/634).

\section{BIBLIOGRAFIA}

1. Chun TW, Stuyver L, Mizell SB, et al. Presence of an inducible HIV-1 latent reservoir during highly active antiretroviral therapy. Proc Natl Acad Sci 1997; 94: 13193-13197.

2. Hughes MD, Johnson VA, Hirsch MS, et al. Monitoring plasma HIV-1 RNA levels in addition to CD4+ lymphocyte count improves assessment of antiretroviral therapeutic response. Ann Intern Med 1997; 126: 929-938.

3. O'Brien WA, Hartigan PM, Daar ES, et al. Changes in plasma HIV RNA levels and CD4+ lymphocyte counts predict both response to antiretroviral therapy and therapeutic failure. Ann Intern Med 1997; 126: 939-945.

4. Perelson AS, Essunger P, Cao Y, et al. Decay characteristics of HIV-1infected compartments during combination therapy. Nature 1997; 387: 188-191.

5. Shain EB, Clemens JM. A new method for robust quantitative and qualitative analysis of real-time PCR. Nucleic Acids Research 2008; 36 (14): e 91.

6. Wong JK, Hezareth M, Gunthard HF, et al. Recovery of replication-competent HIV despite prolonged suppression of plasma viremia. Science 1997; 278:1291-1295. 